\title{
Hybrid Ray Tracing FDTD UWB-Model for Object Recognition
}

\author{
M. Janson, R. Salman, T. Schultze, I. Willms, T. Zwick, W. Wiesbeck
}

\begin{abstract}
In this paper an approach for object recognition by the backscattered UWB signals is presented. This approach is a part of an UWB imaging and localization concept for emergency scenarios, where fire and smoke prevent using optical systems. The proposed recognition method is particulary useful to asses the intactness of the building construction elements. As such elements are usually composed of different materials, often including a metallic core, the recognition of inner structures is of great interest. For effective optimization and application of the recognition approach reference data sets are necessary. These reference data sets are obtained by a hybrid ray tracing/FDTD method, which allows for the simulation of small complex and inhomogenous objects in realsitc indoor scenarios.
\end{abstract}

Index Terms - UWB imaging, IBST, Channel modeling, Ray tracing, FDTD

\section{Introduction}

The Ultra Wideband technology (UWB), first applied by Heinrich Hertz in 1887, has never enjoyed so much interest of researchers and industry as today. The large bandwidth results in a very fine time resolution, which makes UWB rather attractive for radar and imaging applications. One of such applications is imaging of unknown scenarios in case of fire or disasters. Using the UWB sensors, placed on an autonomous robot, a map of the environment can be created. With low frequencies, which propagate easily through most dielectric materials, specific scenario features can be analyzed.

The object recognition method proposed in this paper is part of such an imaging system. The purpose of this method is detection, recognition and classification of hidden and camouflaged objects. This is especially useful for proofing of intactness of the building construction. In this work it is assumed that the object of interest is enclosed in another object of a different material, e. g. a steel girder inside of a concrete pillar. UWB radar measurement data of the considered object are obtained, and compared against the reference data. The task is to recognize both, the outer object, and the enclosed object.

The recognition process is performed on the basis of a previous imaging of the environment, which provides a rough shape and approximate position of objects in a room. For this purpose localization and imaging approaches described in $[1,2,3]$ are used. Furthermore, the permittivity of the outer object needs to be known with suitable accuracy. The permittivity estimation is done by a UWB version of the microwave ellipsometry method described in [4].

The reference data can be obtained either from measurements or from simulations. To enable quick modelling of the composed objects a hybrid ray tracing/finite difference time domain (FDTD) approach is introduced here. This method allows for precise simulation of complex, inhomogeneous objects in realistic scenarios.

\section{Channel model}

The proposed hybrid EM simulator is based on the approach combining UTD and FDTD described in $[5,6]$. In this work the full 3D ray tracing model, described in $[7,8]$, is combined with a FDTD simulator based on $[9,10]$.

The ray tracing channel model uses geometrical optics (GO) and uniform theory of diffraction (UTD). It determines the reflection paths by image theory and diffraction paths by applying Fermat's principle. The good prediction quality of this model has been verified in outdoor scenarios for the frequencies at $2 \mathrm{GHz}$ and $5.2 \mathrm{GHz}[7,8]$. In [11] the extension of the narrow-band model to the UWB band has been described. However, for small objects, which are typically present in indoor scenarios, the ray tracing model is not suitable. Both GO and UTD are asymptotic approaches and assume that the objects in the considered scenario are much larger than the wavelength. If we consider the FCC UWB frequency band $3.1-10.6 \mathrm{GHz}$, with corresponding wavelengths between $\lambda_{3.1 \mathrm{GHz}}=9.7 \mathrm{~cm}$ and $\lambda_{10.6 \mathrm{GHz}}=2.8 \mathrm{~cm}$, some of the objects typically found in the indoor scenarios will not meet this requirement. For some UWB applications, e. g. for a ground penetrating radar or indoor imaging, the lowest frequency can be even below $1 \mathrm{GHz}$. The contributions from objects smaller than a few wavelengths will not be properly modelled by the ray tracing method. To illustrate this, the bistatic scattering coefficient $S_{\mathrm{vv}}$ from a metallic cube with a side length of $6.67 \mathrm{~cm}$ has been simulated with ray tracing (RT) and FDTD at frequencies of $4.5 \mathrm{GHz}$ and $45 \mathrm{GHz}$ (Fig. 1). The incidence angle $\alpha_{\mathrm{i}}$ and the scattering angle $\alpha_{\mathrm{s}}$ are measured in the horizontal plane. The object is illuminated by a plane wave from normal direction $\left(\alpha_{\mathrm{i}}=0\right)$. Vertical polarization of Tx and Rx antennas is used. At the upper frequency of $45 \mathrm{GHz}$ the relative dimensions of the illuminated surface are $10 \lambda \times 10 \lambda$ and the ray tracing result follows the full wave result very well. For the lower frequency of $4.5 \mathrm{GHz}$ the conventional ray tracing overestimates the $S_{v v}$ by almost $10 \mathrm{~dB}$ because the approximations for reflection and diffraction in GO and UTD are not valid for such a small object.

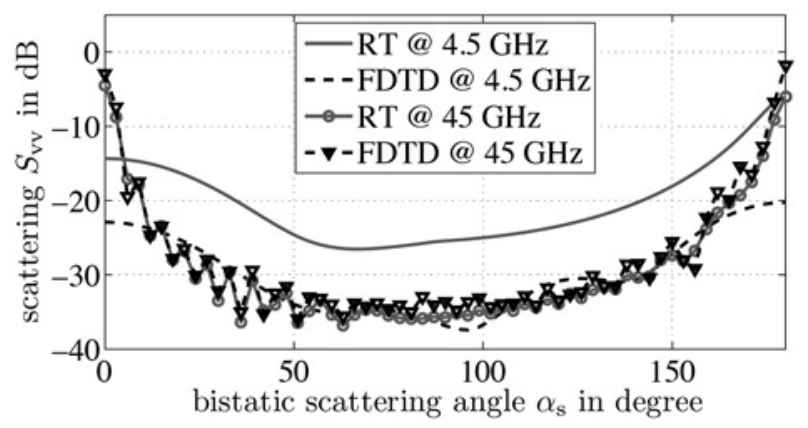

Fig. 1: Bistatic scattering $S_{w}$ from a metallic cube with the side length of $6.67 \mathrm{~cm}$ for normal incidence at vertical polarization $\left(\alpha_{\mathrm{i}}=0\right.$ degree) 
In the following only small objects with line of sight to the transmitter ( $\mathrm{Tx})$ and receiver $(\mathrm{Rx})$ are considered. These objects are identified by preprocessing of the scenario data. For each object found, the incidence and scattering angles for the instantaneous $\mathrm{Tx}$ and $\mathrm{Rx}$ positions are determined. The object is then enclosed in a virtual box. Inside this box a FDTD calculation is run to determine the scattering coefficients. The object is illuminated by a plane wave from the incidence direction and the scattered field is calculated by the scattered field approach [10]. The waveform of the plane wave is Gaussian, thus the scattering coefficients $S_{\mathrm{ij}}$ for all considered frequencies can be calculated in a single run. The convolutional, perfectly matched layer (CPML) is used as an absorbing boundary condition.

The scattered fields are captured on a surface surrounding the object. Using the surface equivalence theorem the electric and magnetic currents at the virtual surface are calculated from the scattered fields and Fourier-transformed. Finally the integration over the whole surface with the free-space Green's function weighting delivers the far field scattering coefficients $S_{\mathrm{ij}}$ in the frequency domain [9].

The obtained coefficients $S_{\mathrm{ij}}$ are then passed back to the ray tracing domain, where the small objects are treated as point scatterers. For each frequency the scattered electric field $\underline{E}_{\mathrm{S}}$ at an observation point at the distance $r$ to the scatterer is calculated from the incident field $\underline{E}_{\mathrm{i}}$ using [12]:

$\underline{E}_{\mathrm{s}}=\frac{e^{-j k_{0} r}}{r}[\underline{\mathbf{S}}] \underline{E_{\mathrm{i}}}, \quad[\underline{\mathbf{S}}]=\left[\begin{array}{ll}\underline{S}_{\mathrm{vv}} & \underline{S}_{\mathrm{vh}} \\ \underline{S}_{\mathrm{hv}} & \underline{S}_{\mathrm{hh}}\end{array}\right]$

Larger objects in the scenario are considered in the ray tracing simulation in a usual way by the GO and the UTD.

\section{Model validation}

For the model validation a small, metallic object with a square cross section of $6 \times 6 \mathrm{~cm}^{2}$ and a height of $80 \mathrm{~cm}$ is considered. The backscattering of the object is measured and simulated by the conventional ray tracing and with the hybrid method for aspect angles from $0^{\circ}$ to $180^{\circ}\left(0^{\circ}=\right.$ normal incidence $)$ in the frequency range from $1 \mathrm{GHz}$ to $4.5 \mathrm{GHz}$. The object has an aluminium surface in order to ensure a strong scattered signal. The measurement is performed with a UWB channel sounder described in [13]. The wavelength at the upper frequency of $4.5 \mathrm{GHz}$ is $6.67 \mathrm{~cm}$, close to the cross section of the object. Although one dimension of the object is larger than the wavelength, an assumption of large flat surfaces is not met for this object.

At each incidence angle $\alpha$ the maximum of the backscattered signal is extracted. Also in this case the backscattered signals simulated with conventional ray tracing are stronger than the signals simulated using hybrid method. For better shape comparison the values are normalized with respect to the maximum of each data series. In Fig. 2a this normalized amplitude $A_{\text {norm }}$ is shown for both simulation methods and the measurement. In the conventional ray tracing simulation reflection and diffraction effects are taken into account, however, the influence of diffraction is small compared to reflections visible at angles $\alpha$ of $0^{\circ}, 90^{\circ}$ and $180^{\circ}$. The result simulated with the hybrid method is more consistent with the measured data.

In Fig. $2 b$ the backscatterd signal in time domain at an incidence angle $\alpha=0^{\circ}$ is shown. Both simulation methods deliver comparable results, hovewer the shape of the signal simulated with the hybrid method follows the measured signal slightly better.

For larger objects the conventional ray tracing solution is

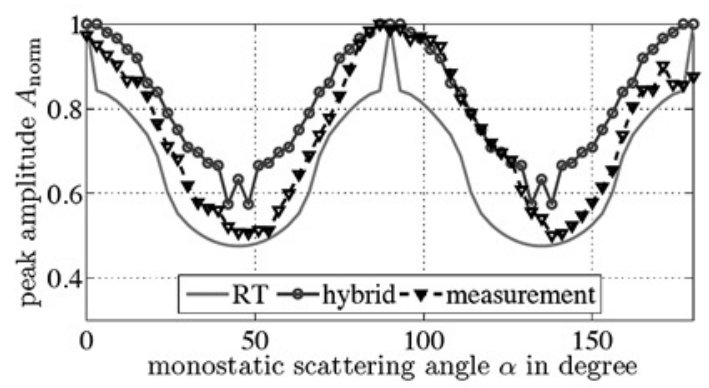

a) normalized peak amplitude $A_{\text {norm }}$ vs. incidence angle $\alpha$

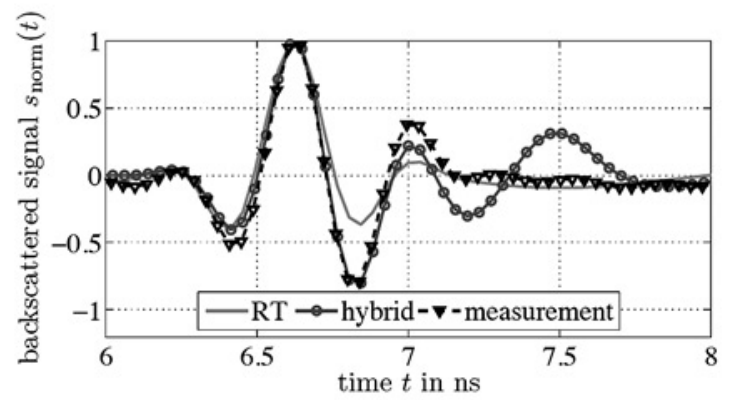

b) backscatterd signal $s_{\text {norm }}(\mathrm{t})$ at incidence angle $\alpha=0$ degree

Fig. 2: Comparison of measured and simulated backscattered signals from the test object

object with a cross section of $30 \times 18 \mathrm{~cm}^{2}$ in the $3.1-10.6 \mathrm{GHz}$ frequency band is presented. In this case the results of conventional ray tracing follow the measurement very well. Furthermore in [14] the hybrid method has been verified in a realistic, complex scenario, showing that if the position of the small objects is in the proximity of the transmitter/receiver, the hybrid method delivers more accurate results than the conventional ray tracing.

Another benefit of this hybrid EM model is its ability to model the transmission through inhomogeneous materials, which cannot be handled by a conventional ray tracing model. This property makes it applicable for the generation of reference data sets used in the image recognition method, described in the following section.

\section{In-wall imaging and object recognition onset}

The tasks of recognizing an outer object and the enclosed object are performed in several steps. The geometrical size and the material properties of the outer object have to be determined and on that basis the unknown shape of the enclosed object is extracted by the dedicated imaging method presented in the following.

Most traditional methods for the reconstruction of object shapes from UWB Radar data rely on coherent summations. A more efficient algorithm is the SEABED onset, achieving an imaging with the outstanding feature to determine the direction of Radar responses, based on changes of the roundtrip times (RTT). The Inverse Boundary Scattering Transform (IBST), proposed in $[15,16]$ as a part of SEABED is given by:

$x\left(x_{\mathrm{w}}\right)=x_{\mathrm{w}}-z_{\mathrm{w}} \cdot \mathrm{d} z_{\mathrm{w}} / \mathrm{d} x_{\mathrm{w}}$

$z\left(x_{\mathrm{w}}\right)=z_{\mathrm{w}} \cdot \sqrt{1-\left(\mathrm{d} z_{\mathrm{w}} / \mathrm{d} x_{\mathrm{w}}\right)^{2}}$

It transforms the quasi-wavefronts, obtained from radargrams, into curves describing the object contour. The coordinates $(x, z)$ represent a reflection point at the boundary of a $2 \mathrm{D}$ object. The variable $z_{\mathrm{w}}$ is the distance to the object at the antenna position $\left(x_{\mathrm{w}}, 0\right)$. The IBST originates from the inter- 


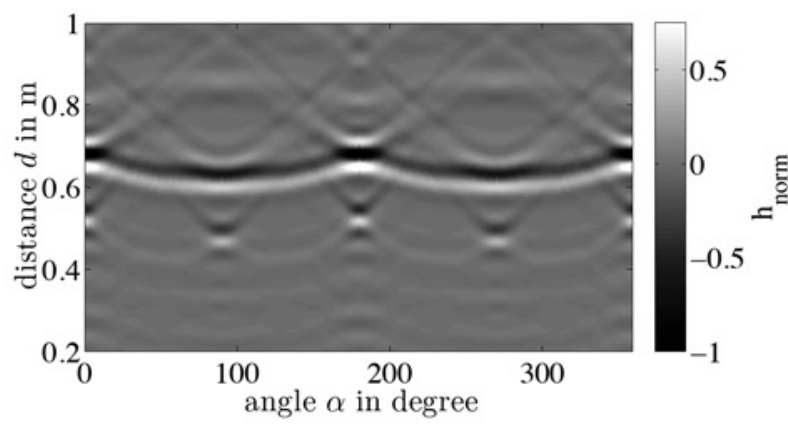

a) hybrid ray tracing/FDTD simulation

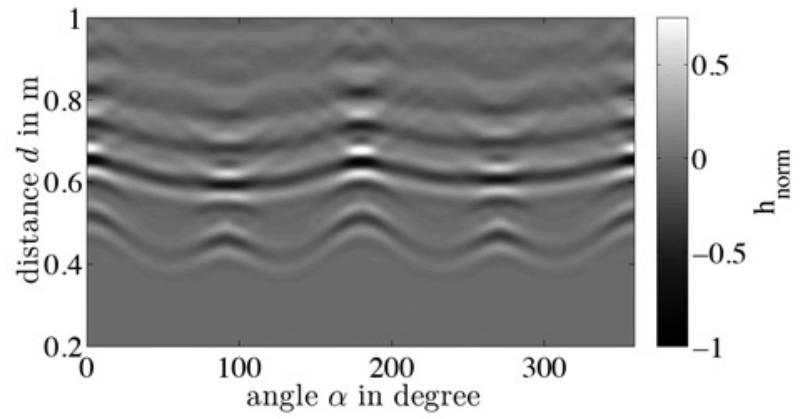

b) measurement

Fig. 3: Measured and simulated radargram of an embedded object with rectangular cross-section; for the related data see Fig. 4

pretation of a quasi-wavefront such that each reflection point on the object boundary must be located on a circle with a radius $z_{\mathrm{w}}$, centred at the antenna position. One onset to reconstruct the object boundary is to compute the envelope of the family of circles with centers at the antenna position and with radii $z_{\mathrm{w}}\left(x_{\mathrm{w}}\right)$ according to the RTT of the Radar pulses.

Although this approach has been extended to bi-static configurations, 3D objects and non-planar tracks, up to now it was not applied to the reconstruction of objects hidden and enclosed in a dielectric outer body. The main problem is that refraction at a dielectric boundary causes changes to the spherical shape of the wavefronts outside of objects. This fact contradicts the assumption of IBST that the object boundary should lay on an envelope of circles.

In [17] a method for describing refracted wavefronts in a medium is proposed. It is derived for an antenna moving at a certain distance in front of a dielectric medium like a thick wall. This method is based on computing a hypothetical parent wavefront, which is an auxiliary construct according to the hyperbola described by:

$\frac{z_{\mathrm{h}}^{2}}{a\left(z_{\mathrm{w}}\right)^{2}}-\frac{\left(x_{\mathrm{h}}-x_{\mathrm{w}}\right)^{2}}{b\left(z_{\mathrm{w}}\right)^{2}}=1$ with

$a=z_{\mathrm{W}} / n, \quad b=a \sqrt{n^{2}-1}, \quad n=\operatorname{Re}\left\{\sqrt{\varepsilon_{\mathrm{t}} / \varepsilon_{\mathrm{i}}}\right\}$

where $n$ is the refractive index, $\varepsilon_{t}$ being the relative permittivity of the transmitting medium, and $\varepsilon_{\mathrm{i}}$ the relative permittivity of the incident medium. $\left(x_{\mathrm{W}}, z_{\mathrm{W}}\right)$ here represent the antenna position. To obtain the real wavefront $\left(x_{\mathrm{a}}, z_{\mathrm{a}}\right)$ of a wave with an RTT of $t_{\mathrm{x}}$ each point of the parent wavefront is shifted by a distance of

$p=0.5 \cdot t_{\mathrm{x}} \cdot c_{0} / \sqrt{\varepsilon_{t}}$

in directions perpendicular to the parent wavefront curve. The curves described by equations (3) and (4) represent true waveforms inside of the dielectric half-space but no inversion formula is known for obtaining the enclosed object surface points $(x, z)$. In [17] so-called best fit hyperbolas according to:

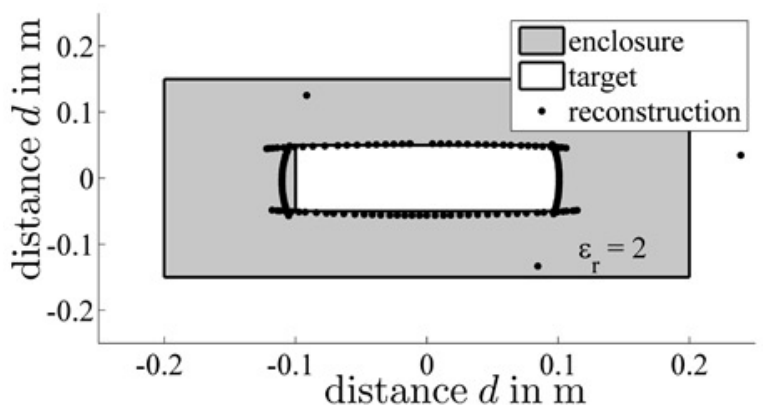

Fig. 4: Measured image of a rectangular metallic object $\left(10 \times 20 \mathrm{~cm}^{2}\right)$, embedded in gas concrete with a relative permittivity of $\varepsilon_{r}=2$, and a size of $30 \times 40 \mathrm{~cm}^{2}$

$F=\frac{\left(z-z_{\mathrm{a} 0}\right)^{2}}{u^{2}}-\frac{\left(x-x_{\mathrm{w}}\right)^{2}}{v^{2}}-1$ with $z_{\mathrm{a} 0}=-p\left(x_{\mathrm{w}}\right) \frac{n}{\sqrt{n^{2}-1}}$,

$u=\frac{z_{\mathrm{w}}}{n}-p\left(x_{\mathrm{w}}\right)-z_{a 0}$ and $v=u \sqrt{n^{2}-1}$

are described. Using these hyperbolas the surface points can be found by determining the envelope of the family of all wavefronts according to [15] by the additional condition $\mathrm{d} F / \mathrm{dx}_{\mathrm{w}}=0$.

In the measurements described in the next chapter, for the outer wall limited rectangular plates with straight surface segments are used. This simplifies the measurements and allows to analyse the applicability of the extended IBST algorithm to non-wall objects like a pillar including a steel girder. On the other hand artifacts of the inner object are appearing for antenna positions close to corners of these plates. Therefore measurement data at these antenna positions have to be discarded in the imaging process.

\section{Measurement setup and results}

In order to verify the correct operation of the proposed UWB in-wall imaging algorithm a series of experiments was performed. Initially the wavefront extraction and extended IBST algorithms were applied to the hybrid ray tracing/FDTD simulated radargram, which was afterwards validated by measurements. Figure 3 shows the simulated and measured radargrams, obtained from an enclosed metallic rectangular object of $20 \mathrm{~cm}$ by $10 \mathrm{~cm}$. This object was embedded in the centre of a sand-lime brick cover with an rectangular cross section of $30 \mathrm{~cm}$ by $40 \mathrm{~cm}$. The distance $d$ between the antenna and the centre of this combined object was $65 \mathrm{~cm}$. Fig. 3 shows that measurement and simulation agree very well. Especially the multiple reflections are visible in both cases quite well. Figure 4 shows the corresponding image of the enclosed object obtained from the measurement data.

Several similar rectangular objects were built with the same size as used in the simulations. The size of the outer blocks is $30 \times 40 \mathrm{~cm}^{2}$, the material is either sand-lime brick with a relative permittivity of $\varepsilon_{\mathrm{r}}=4$, gas concrete with $\varepsilon_{\mathrm{r}}=2$, or plaster with $\varepsilon_{\mathrm{r}}=4$. A set of embedded metallic objects were a cylinder, a square cross-section bar and a rectangular crosssection bar, both for simulations and measurements.

The object recognition task is performed on the basis of the binary Radar images showing single points for the inner objects surface obtained by the extended IBST algorithm. The IBST algorithm is applied to both, simulation results (for obtaining reference data) and the measurement data. These images are postprocessed using two object recognition algorithms, i.e. the moment invariant method and polar Fourier descriptors, explained in detail in [4] and [18].

According to these object recognition onsets, the object recognition algorithms were applied to images of all 3 
enclosed objects. The obtained results shown in (6) are given by the matrices $\mathrm{M}_{\text {Moment }}$ and $\mathrm{M}_{\text {Fourier }}$ having the dimension $A \times \mathrm{K}$, whereby $A$ and $\mathrm{K}$ are the identification numbers of measured objects and reference objects, respectively.

$\mathrm{M}_{\text {Moment }}=\left(\begin{array}{ccc}5.42 & 6.20 & 8.11 \\ 6.48 & 6.50 & 7.24 \\ 6.90 & 7.34 & 5.59\end{array}\right)$

$\mathrm{M}_{\text {Fourier }}=\left(\begin{array}{ccc}0.074 & 0.24 & 0.13 \\ 0.28 & 0.10 & 0.18 \\ 0.08 & 0.25 & 0.01\end{array}\right) \cdot 10^{-4}$.

Each row of the matrix $\mathrm{M}_{\text {Moment }}$ shows the similarity function values for one measured object $(A=K=1$ for the cylinder, $A=K=2$ for the rectangular bar and $A=K=3$ for the square bar). The polar Fourier descriptors use a least mean square error as a classifier. The matrix elements describe the similarity between the measured and reference data. The lower the value is, the higher is the similarity and equals 0 for identical objects. The moment invariant method recognises both the cylinder and the square, but slightly fails in the recognition of the rectangular bar. The polar fourier descriptors recognise all object by far correctly and shows much more stable behaviour than the moment invariant method.

\section{Conclusions}

The presented hybrid ray tracing/FDTD channel model is able to predict the scattering from small objects considerably better than conventional ray tracing. Also the inhomogeneities inside an object can be considered in the simulations. This property makes it useful for creating reference data sets for the proposed object recognition approach. With the object recognition method the shape of the structures, enclosed in objects with dielectrical properties, can be estimated with a good precision, provided that an estimation of the shape and material parameters of the outer object is available.

\section{References}

[1] R. Thomä, O. Hirsch, J. Sachs, R. Zetik, "UWB Sensor Networks for Position Location and Imaging of Objects and Environments," Proc. 2nd European Conf. on Antennas and Propagation EuCAP 2007, pp. 1-9, Edinburgh, UK, Nov. 2007.

[2] R. Zetik, J. Sachs, R. Thomä, "Imaging of Propagation Environment by UWB Channel Sounding," Proc. XXVIIIth General Assembly URSI, New Delhi, India, Oct. 2005.

[3] J. Seitz, M. Schaub, O. Hirsch, R. Zetik, T. Deißler, R. Thomä, J Thielecke, "UWB Feature Localization for Imaging", Proc. IEEE Int. Conf. on Ultra-Wideband, ICUWB 2008, Hannover, Germany, Sept. 2008

[4] R. Salman, Th. Schultze, I. Willms, "UWB Material Characterisation and Object Recognition with Applications in Fire and Security," Proc. IEEE Int. Conf. on Ultra-Wideband, ICUWB 2008, Hannover Germany, Sept. 2008.

[5] S. Reynaud, Y. Cocheril, R. Vauzelle, A. Reineix, L. Aveneau, and C. Guiffaut, "Influence of an accurate Environment Description for the
Indoor Propagation Channel Modelling”, Proc. Eur. Conf. on Wireless Technology, Paris, France, Oct. 2005.

[6] S. Reynaud, Y. Cocheril, R. Vauzelle, C. Guiffaut, and A. Reineix, "Hybrid FDTD/UTD Indoor Channel Modeling. Application to Wifi Transmission Systems", Proc. 64th IEEE Veh. Technol. Conf., VTC2006 Fall, pp. 1-5, Montreal, Canada, Sept. 2006.

[7] T. Fügen, J. Maurer, T. Kayser, and W. Wiesbeck, "Capability of 3-D Ray Tracing for Defining Parameter Sets for the Specification of Future Mobile Communications Systems," IEEE Trans. Antennas Propag., vol. 54, no. 11, pp. 3125-3137, Nov. 2006.

[8] J. Maurer, "Strahlenoptisches Kanalmodell für die Fahrzeug-Fahrzeug-Funkkommunikation," (in German) Ph.D. dissertation, Univ. of Karlsruhe, Karlsruhe, Germany, 2005.

[9] A. Taflove and S. C. Hagness, ,Computational Electrodynamics: The Finite-Difference Time-Domain Method,“ 3rd ed. Norwood, MA, Artech House, 2005.

[10] K.S. Kunz and R.J. Luebbers ,The Finite Difference Time Domain Method for Electromagnetics," CRC Press, 1993.

[11] C. Sturm, W. Sörgel, T. Kayser, and W. Wiesbeck, „Deterministic UWB Wave Propagation Modeling for Localization Applications based on 3D Ray Tracing," Proc. IEEE MTT-S Int. Microw.Symposium, pp. 2003-2006, San Francisco, California, June 2006.

[12] N. Geng and W. Wiesbeck, ,Planungsmethoden für die Mobilkommunikation," (in German). Berlin, Heidelberg Springer, 1998.

[13] J. Sachs, R. Thomä, U. Schultheiss, R. Zetik, J. Dvoracek, M. Wolf, "Real-time Ultra-Wideband Channel Sounder," Proc. XXVIIth General Assembly URSI, Maastricht, Netherlands, Aug. 2002.

[14] M. Porebska, T. Kayser, and W. Wiesbeck, "Verification of a Hybrid Ray-Tracing/FDTD Model for Indoor Ultra-Wideband Channels," Proc. Eur. Conf. on Wireless Technology, Munich, Germany, Oct. 2007.

[15] T. Sakamoto, T. Sato, "A Target Shape Estimation Algorithm for Pulse Radar Systems based on Boundary Scattering Transform," IEICE Trans. Commun. vol. E87-B, no. 5, pp. 1357-1365, May 2004.

[16] S. Kidera, T. Sakamoto, and T. Sato, "A Robust and fast Imaging Algorithm with an Envelope of Circles for UWB Pulse Radars," IEICE Trans Commun, vol. E90-B, no.7, pp. 1801-1809, Jul. 2007.

[17] C. Rappaport, "A Simple Approximation of Transmitted Wavefront Shape from Point Sources above lossy Half Spaces," Proc. Geoscience and Remote Sensing Symposium, 2004, vol. 1, pp. 421-424, Anchorage, Alaska, USA, Sept. 2004.

[18] R. Salman, Th. Schultze, M. Janson, W.Wiesbeck, I. Willms, "Robust UWB Radar Object Recognition," IEEE Int. RF and Microw. Conf., RFM 2008, Kuala Lumpur, Dec. 2008.

\section{First Author}

Malgorzata Janson

Institut für Hochfrequenztechnik und Elektronik

Universität Karlsruhe (TH)

Karlsruhe, Germany

E-mail: malgorzata.janson@kit.edu

Other authors

Rahmi Salman, Thorsten Schultze, Ingolf Willms

Fachgebiet Nachrichtentechnische Systeme

Universität Duisburg-Essen

Duisburg, Germany

Thomas Zwick, Werner Wiesbeck

Institut für Hochfrequenztechnik und Elektronik

Universität Karlsruhe (TH)

Karlsruhe, Germany

This work has been supported by DFG within the priority programme 1202 "UWB Radio Technologies for Communication, Localization and Sensor Technology (UKoLos)". We would also like to thank our partners at the Technische Universität Ilmenau and the Friedrich-Alexander-Universität Erlangen-Nürnberg for the excellent co-operation in the project on "Cooperative Localisation and Object Recognition in Autonomous UWB Sensor Networks". 